\title{
Maximum Efficiency STEM Phase Contrast Imaging
}

\author{
Timothy J. Pennycook ${ }^{1,2}$, Andrew R. Lupini ${ }^{3}$, Lewys Jones ${ }^{1}$ and Peter D. Nellist ${ }^{1,2}$ \\ 1. Department of Materials, University of Oxford, Parks Road, Oxford OX1 3PH, UK \\ 2. SuperSTEM Laboratory, Daresbury, WA4 4AD, UK \\ 3. Oak Ridge National Laboratory, Oak Ridge, TN 37831, USA
}

Various bright-field (BF) imaging modes are available in scanning transmission electron microscopy (STEM). The mode equivalent to phase contrast imaging in conventional high-resolution transmission electron microscopy (TEM) involves a detector about an order of magnitude smaller than the convergence angle of the probe. This means a large proportion of the incident electrons go to waste as most of the bright field disk is left unused. The annular bright-field (ABF) mode of imaging uses a larger area of the bright-field disk and has been successful in revealing columns of low-Z elements. However complications in interpretation remain as contrast in ABF images can be generated through both channelling and phase-contrast effects arrising from residual aberrations [1]. In the differental phase-contrast (DPC) mode of imaging, almost the entire bright field disk can be used. In DPC multiple detectors are placed in the BF disk, typically in a four quadrant configuration. To generate contrast, the signal from oposing quadrants is subtracted, providing sensitivity to shifts in the beam caused by electrostatic or magnetic fields in the sample [2].

An alternative approach that provides greater flexability is to record the entire BF disk with a pixelated detector [3]. This allows one to choose the BF mode after acquiring the data, and even compare different modes from the same scan. However a more sophisticated method can be used with a pixelated detector. Rodenburg et al. used such a detector to allow STEM ptychography to retrieve the phase of the object function [4]. Their work was focused on overcoming the diffraction limit to imaging resolution. Now with aberration correction enabling routine atomic resolution imaging, the limiting parameter is often beam induced damage rather than the spatial resolution of the microscope. With beam sensitive materials one desires to extract the maximum amount of information per electron. We have therefore adapted the technque developed by Rodenburg et al to provide maximum efficiency phase contrast imaging.

Figure 2 shows an example of a phase-contrast image created with STEM ptychography. A ronchigram was recorded at each probe position simultaneously with the annular dark field (ADF) intensity. The roughly 4000 Ronchigrams were then Fourier transformed with respect to probe position. By extracting information only from regions of Fourier space in which the wavefunctions are transferred we reject noise and obtain the best possible statistics on the phase and amplitude of each spacial frequency. We can then interfere the spatial frequencies to form the highest efficiency phase contrast image possible in STEM.

Compared to a TEM the method has the advantage of offering phase contrast images at the full resolution of the microscope without the need for aberrations. Through combining with simultaneous ADF imaging we maximize the information by collecting signal over essentially all available scattering angles. ADF imaging also facilitates the interpretation of the images and provides access to low-spatial frequencies, which are not transferred in convention BF imaging. We will present further experimental developments, data and theoretical calculations to investigate the optimal configuration for the maximum phase sensitivity with the lowest dose [5]. 


\section{References:}

[1] S. Lee et al. Ultramicroscopy, 125, (2013), p. 43.

[2] N. Shibata et al, Nature Physics, 8, (2012), 611.

[3] K. Kimoto and K. Ishizuka, Ultramicroscopy, 111, (2011), p. 1111.

[4] J. M. Rodenburg, B. C. McCallum and P. D. Nellist, Ultramicroscopy, 48, (1993), p. 303.

[5] This research was supported by the EPSRC through the UK National Facility for Aberration-

Corrected STEM (SuperSTEM). Research at Oak Ridge National Laboratory was sponsored by the US Department of Energy, Office of Science, Materials Sciences and Engineering Division.
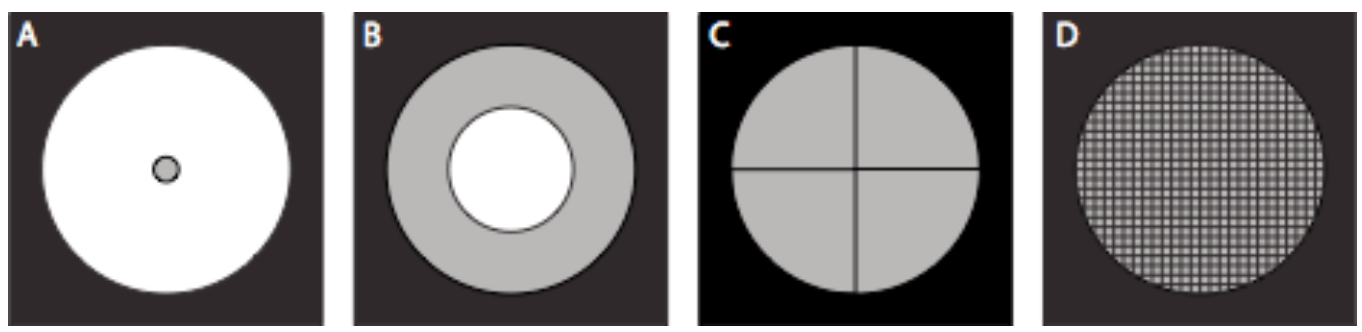

Figure 1. Schematic showing the geometries of a conventional BF detector (A), an ABF detector (B), the quadrant detectors used in DPC (C), and recording the entire BF disk with a pixelated detector (D). The convergence angle is indicated by the inner bound of the black outer region. Detectors are colored grey with black edges.
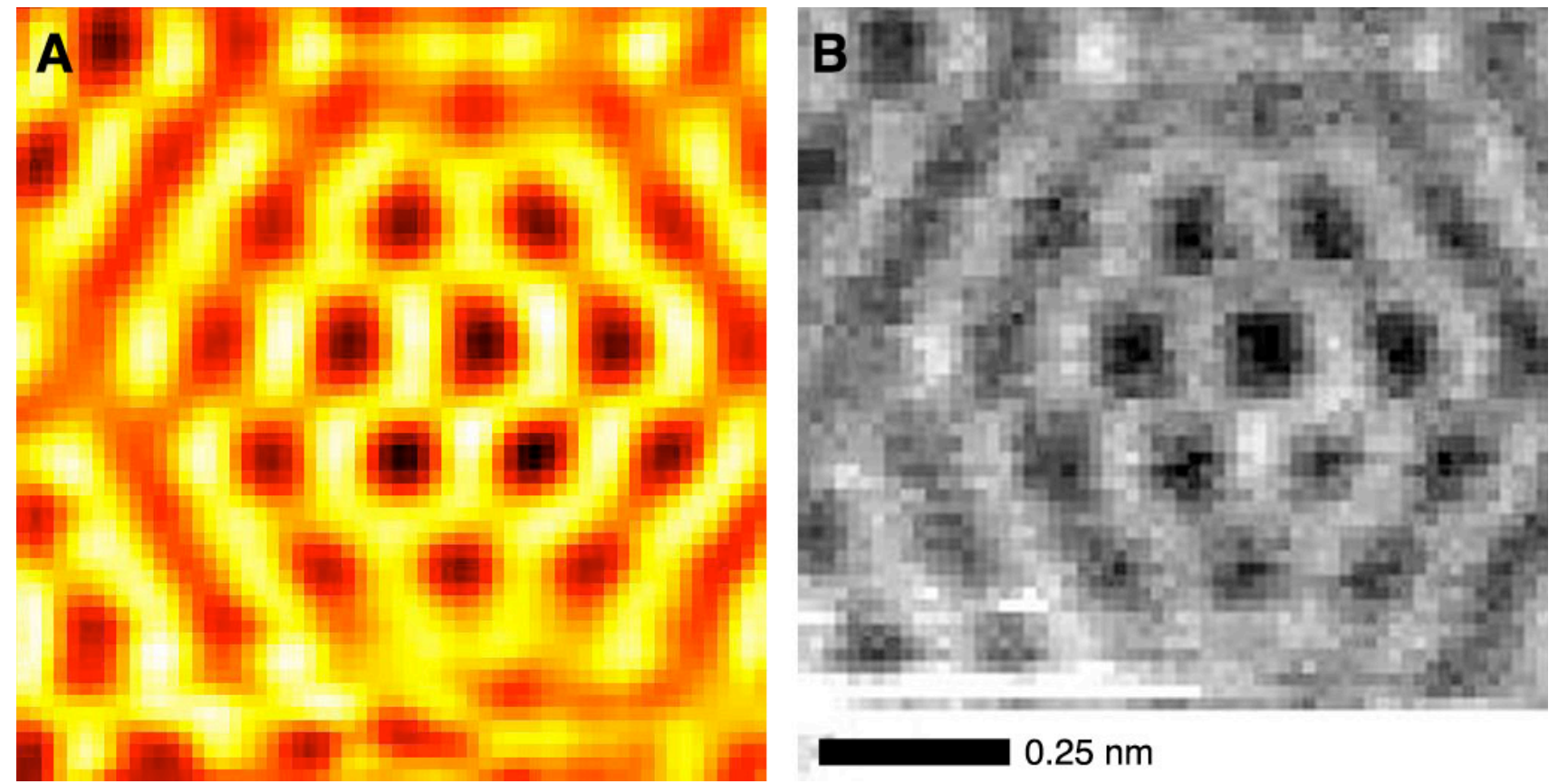

Figure 2. A phase-contrast image constructed using ptycography (A) and a simultaneously acquired ADF image (B) of a graphene bilayer. The saturated region at the bottom of $\mathrm{B}$ is due to the onset of contamination. The phase-contrast image appears noticeably less noisy than the ADF image. 